\title{
National Differences In Investors' Responses To Corruption: An International Comparison
}

Mohsin Habib, (E-mail:mohsin_h@hotmail.com), University of Massachusetts-Boston Leon Zurawicki, (E-mail:leon.zurawicki@umb.edu), University of Massachusetts-Boston

\begin{abstract}
This paper looks into the differences in responses to corruption by foreign investors. Statistical analysis highlights such differences for 17 major investing countries. Those differences appear to be related to home countries' own level of corruption, their size and experience in foreign investing.
\end{abstract}

\section{Introduction}

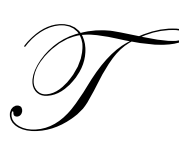

he question of how foreign investors from individual home countries respond to local corruption in the host market is certainly intriguing albeit not fully researched one. On the one hand, recent studies confirmed the overall negative reaction to corruption (Wei, 2000, Drabek and Paine, 1999, Habib and Zurawicki, 2002). On the other, even intuitively it would be hard to expect that all the investors display the same degree of sensitivity. Familiarity with the level of the characteristics of corruption in the target markets has been identified as a relevant factor affecting the foreign investors' decisions according to Habib and Zurawicki, 2002.

To date, there have been very few studies focusing on possible differences in the responses of foreign direct investors from individual countries to the corruption in target markets. Academic articles which addressed this particular issue rather on the margin of the more general questions discussed, actually found no significant differences between the investors' countries despite their varying legislature addressing foreign corruption (Hines, 1995, Wei, 2000).

However, while examining a similar issue with respect to the international trade, Lambsdorff (1998) concluded that certain countries might have an advantage over others in the import markets perceived to be corrupt. This would imply that the taxing impact of corruption does not have to be uniform and ultimately is a function of, among other things, the exporters' differentiated propensity to offer bribes.

\section{Propositions}

The above findings motivated the authors to address anew the issue of how corruption affects the incoming foreign direct investments (FDI) originating in individual home countries. In this paper, the following propositions were developed:

P1. All other things being equal, investors from countries perceived as relatively corrupt are less hampered by perceived corruption in the home countries than the investors from countries perceived as relatively less corrupt.

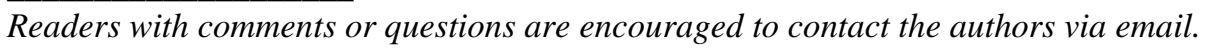


P2. All other things being equal, investors from countries which are latecomers to the FDI activities are less impeded by perceived corruption in the host countries than the investors from countries which established international presence early on. (as the re-investment accounts for a substantial portion of FDI).

P3. All other things being equal, investors from larger countries are more sensitive to perceived corruption in the home countries than the investors from smaller countries. This would reflect a greater absorption capacity for local investment in the large home countries.

\section{Data and Methodology}

\subsection{Sample}

The study included the largest possible number of host countries in order to obtain a broader perspective on FDI and corruption. The statistics on aggregate bilateral FDI flows are analyzed in conjunction with key observational variables. Most data for the sample was obtained from the OECD International Direct Investment Statistics Yearbook (OECD, 2000). The bilateral flows of FDI between the largest investor-countries and the recipients of their FDI were recalculated in the US dollars for the three last years (1996-1998) for which the data was available. The following 17 home countries were included in the analysis: Australia, Austria, Denmark, Finland, France, Germany, Italy, Japan, Korea, the Netherlands, Norway, Portugal, Spain, Sweden, Switzerland, UK and the USA. The selection of home countries is meant to provide a fair representation of the Triad, while incorporating countries with markedly varying levels of Corruption Perception Index (CPI), the corruption measure produced by Transparency International. This is very important, as based on the methodology applied by Transparency International, relatively small differences in CPI obtained for individual countries are not statistically significant. Depending on the home country, between 50 and 70 target host countries were identified for each of the 3 years. The home and the host countries considered represent the whole international spectrum, including developed and developing economies as well as the transition economies of Eastern Europe.

\subsection{Measurements}

The OLS regression model was applied and ran separately for all the home countries--17 altogether. For each home country, two models are analyzed, both of them using the log of FDI as the dependent variable. FDI is the annual inflow of foreign direct investment from the home country to the host country. Log of FDI was used in the analysis to make the distributions nearly normal and the error term homoscedastic. The first model includes the control variables and CPI. The second model replaced CPI with the absolute difference in CPI. The key independent variables are corruption (corruption perception index or CPI) and absolute difference in corruption (Abs. Diff. in CPI) between the host and the home country. In this study, the corruption measure collected by Transparency International (1999) is used and alternatively, a derived measure of the absolute difference in the perceived level of corruption between the home and the host countries was calculated by the authors.

The key independent variables used in the regression - the same in Model 1 and 2-were selected based on the determinants of FDI as suggested in the literature (Ensign, 1996).

The majority of the results offer support for the propositions formulated earlier-refer to Table 1 . The existence of nuances in the general pattern uncovered is admissible in view of possible country-specific factors. Interestingly, in many instances Model 1 and 2 rendered very similar results in terms of the value of the coefficients characterizing the impact of the perceived corruption level and the absolute difference in the perceived corruption levels upon foreign direct investment. This could be due to the fact that on the average the home countries are perceived significantly less corrupt than the majority of the target countries. 


\section{References}

1. Drabek, Z. \& W. Payne. 1999. "The impact of transparency on foreign direct investment". Staff Working Paper ERAD-99-02, Geneva, World Trade Organization.

2. Ensign, P. 1996. "An examination of foreign direct investment theories and the multinational firm: A business/economics perspective". In M. Green \& R. McNaughton, editors, The Location of Foreign Direct Investment, Avebury: Aldershot.

3. Habib, and L. Zurawicki. 2002. "Corruption and Foreign Direct Investment", Journal of International Business Studies,

4. Hines, J. 1995. "Forbidden payments: Foreign bribery and American business after 1977". Working Paper 5266, National Bureau of Economic Research, Cambridge.

5. Lambsdorff, G. (1998). "An Empirical Investigation of Bribery in International Trade”. European Journal of Development Research, 11 (1), 40-59.

6. $\quad$ OECD. 2000. International Direct Investment Statistics Yearbook 1999, Paris.

7. TI. 1999. www.transparency.org/documents/cpi.

8. Wei, S. 2000. "How taxing is corruption on international investors?" The Review of Economics and Statistics 82(4): 1-12.

9. Wheeler, D. \& A. Mody. 1992. "International investment location decisions: The case of U.S. firms". Journal of International Economics, 33: 57-76.

Notes 
Table 1. Regression coefficients for individual investor countries.

\begin{tabular}{|c|c|c|c|c|c|c|c|c|c|c|}
\hline & \multicolumn{2}{|c|}{ Australia } & \multicolumn{2}{|c|}{ Austria } & \multicolumn{2}{|c|}{ Denmark } & \multicolumn{2}{|c|}{ Finland } & \multicolumn{2}{|c|}{ France } \\
\hline & Model 1 & Model 2 & Model 1 & Model 2 & Model 1 & Model 2 & Model 1 & Model 2 & Model 1 & Model 2 \\
\hline \multirow[b]{2}{*}{ Constant } & $-10.89 *$ & -5.773 & $-5025 \dagger$ & 3.38 & $-4.044 \dagger$ & -0.49 & $-10.747 * *$ & -4.473 & $-9.312 * *$ & $-8.981 * *$ \\
\hline & $(5.136)$ & $(5.391)$ & $(3.578)$ & $(3.924)$ & $(2.171)$ & $(2.303)$ & $(3.543)$ & $(3.768)$ & (3.698) & $(4.003)$ \\
\hline \multirow[b]{2}{*}{ Econ. Ties } & $0.185^{*}$ & $0.168 *$ & $0.392 * * *$ & $0.356 * * *$ & $0.229 * *$ & $0.229 * *$ & -0.067 & -0.067 & 0.088 & $0.124 * *$ \\
\hline & $(0.513)$ & $(0.505)$ & $(0.659)$ & $(0.657)$ & $(0.381)$ & $(0.38)$ & $(0.489)$ & $(0.486)$ & $(0.563)$ & $(0.571)$ \\
\hline \multirow[b]{2}{*}{ LogDist } & -0.051 & -0.061 & -0.111 & -0.114 & $-0.248 * * *$ & $-0.249 * * *$ & $-0.289 * * *$ & $-0.288 * * *$ & $-0.328 * * *$ & $-0.337 * * *$ \\
\hline & $(1.004)$ & $(0.99)$ & $(0.555)$ & $(0.548)$ & $(0.394)$ & $(0.393)$ & $(0.468)$ & $(0.466)$ & $(0.517)$ & $(0.537)$ \\
\hline \multirow[b]{2}{*}{ LOGPOP } & $0.283 * *$ & $0.290 * *$ & $0.205 * * *$ & $0.160 * *$ & $0.238 * * *$ & $0.237 * * *$ & $0.294 * * *$ & $0.290 * * *$ & $0.236 * * *$ & $0.199 * * *$ \\
\hline & $(0.341)$ & $(0.332)$ & $(0.316)$ & $(0.307)$ & $(0.191)$ & $(0.191)$ & $(0.323)$ & $(0.32)$ & $(0.336)$ & $(0.335)$ \\
\hline \multirow[b]{2}{*}{ TRADE/GDP } & -0.075 & -0.072 & -0.009 & -0.035 & -0.08 & -0.081 & -0.094 & $-0.097 \dagger$ & $-0.122 * *$ & $-0.139 * *$ \\
\hline & $(0.516)$ & $(0.501)$ & $(0.365)$ & $(0.358)$ & $(0.214)$ & $(0.214)$ & $(0.366)$ & $(0.363)$ & $(0.386)$ & $(0.397)$ \\
\hline \multirow[b]{2}{*}{ Polit. Risk } & $0.267 *$ & $0.195 *$ & -0.104 & -0.057 & $0.185^{*}$ & $0.186^{*}$ & $0.329 * * *$ & $0.325 * * *$ & $0.326 * * *$ & $0.515^{* * * *}$ \\
\hline & $(0.027)$ & $(0.026)$ & $(0.028)$ & $(0.026)$ & $(0.017)$ & $(0.017)$ & $(0.029)$ & $(0.028)$ & $(0.028)$ & $(0.023)$ \\
\hline \multirow[b]{2}{*}{ CPI } & $0.314 * *$ & & $0.536 * * *$ & & $0.370 * * *$ & & $0.410 * * *$ & & $0.302 * * *$ & \\
\hline & $(0.13)$ & & $(0.124)$ & & $(0.077)$ & & $(0.138)$ & & $(0.132)$ & \\
\hline \multirow[b]{2}{*}{ Abs. Dif CPI } & & $-0.403 * *$ & & $-0.517 * * *$ & & -0.37 & & $-0.416 * * *$ & & -0.053 \\
\hline & & $(0.144)$ & & $(0.166)$ & & $(0.077)$ & & $(0.136)$ & & $(0.179)$ \\
\hline \begin{tabular}{|l} 
Adj. R $^{2}$ \\
\end{tabular} & 0.303 & 0.323 & 0.553 & 0.564 & 0.263 & 0.623 & 0.619 & 0.622 & 0.658 & 0.629 \\
\hline $\mathbf{N}$ & 161 & 161 & 170 & 170 & 166 & 166 & 158 & 158 & 172 & 173 \\
\hline $\mathbf{F}$ & $13.099 * * *$ & $14.273 * * *$ & $37.312 * * *$ & $38.894 * * *$ & $48.339 * * *$ & $48.419 * * *$ & $45.448 * * *$ & $45.950 * * *$ & $58.135 * * *$ & $51.585^{* * *}$ \\
\hline
\end{tabular}


Table 1 (continued)

\begin{tabular}{|c|c|c|c|c|c|c|c|c|c|c|}
\hline & \multicolumn{2}{|c|}{ Germany } & \multicolumn{2}{|c|}{ Italy } & \multicolumn{2}{|c|}{ Japan } & \multicolumn{2}{|c|}{ Korea } & \multicolumn{2}{|c|}{ Netherlands } \\
\hline & Model 1 & Model 2 & Model 1 & Model 2 & Model 1 & Model 2 & Model 1 & Model 2 & Model 1 & Model 2 \\
\hline \multirow[b]{2}{*}{ Constant } & $-27.952 * * *$ & $-22.947 * * *$ & $-23.308 * * *$ & $-24.703 * * *$ & $-43.539 * * *$ & $-42.892 * * *$ & $-42.525 * * *$ & $-41.105 * * *$ & $-8.483 * *$ & -3.841 \\
\hline & $(3.706)$ & (4.107) & $(3.279)$ & $(3.268)$ & $(6.975)$ & $(7.273)$ & $(6.305)$ & $(6.257)$ & $(3.775)$ & $(4.066)$ \\
\hline \multirow[b]{2}{*}{ Econ. Ties } & $0.426 * * *$ & $0.418 * * *$ & $0.114 \dagger$ & $0.130 \dagger$ & $0.219 * *$ & $0.230 * * *$ & 0.325 & $0.303 * * *$ & $0.127 *$ & $0.129 *$ \\
\hline & $(0.693)$ & $(0.705)$ & $(0.517)$ & $(0.523)$ & $(0.768)$ & $(0.784)$ & $(0.777)$ & $(0.774)$ & $(0.563)$ & $(0.556)$ \\
\hline \multirow[b]{2}{*}{ LogDist } & -0.067 & -0.067 & $-0.212 * * *$ & $-0.216 * * *$ & 0.059 & 0.052 & $0.135 \dagger$ & 0.107 & $-0.415 * * *$ & $-0.415 * * *$ \\
\hline & $(0.603)$ & $(0.611)$ & $(0.459)$ & $(0.473)$ & $(1.249)$ & $(1.281)$ & $(1.078)$ & $(1.076)$ & $(0.53)$ & $(0.526)$ \\
\hline \multirow[b]{2}{*}{ LOGPOP } & $0.490 * * *$ & $0.469 * * *$ & $0.571 * * *$ & $0.561 * * *$ & $0.685 * * *$ & $0.645^{* * *}$ & $0.645 * * *$ & $0.630 * * *$ & $0.312 * * *$ & $0.311^{* * *}$ \\
\hline & $(0.324)$ & $(0.323)$ & $(0.305)$ & $(0.305)$ & $(0.411)$ & $(0.412)$ & $(0.408)$ & $(0.401)$ & $(0.341)$ & $(0.336)$ \\
\hline \multirow[b]{2}{*}{ TRADE/GDP } & 0.04 & 0.022 & $.106^{*}$ & $0.105 \dagger$ & $0.162 * *$ & $0.129 *$ & 0.059 & 0.056 & -0.064 & -0.067 \\
\hline & $(0.403)$ & $(0.405)$ & $(0.357)$ & $(0.361)$ & $(0.446)$ & $(0.453)$ & $(0.414)$ & $(0.408)$ & $(0.383)$ & $(0.38)$ \\
\hline \multirow[b]{2}{*}{ Polit. Risk } & $0.192 * *$ & $0.234 * *$ & $0.415 * * *$ & $0.508 * * *$ & $0.291 * *$ & $0.468 * * *$ & $0.304 * * *$ & $0.359 * * *$ & $0.288 * * *$ & $0.265^{* *}$ \\
\hline & $(0.028)$ & $(0.028)$ & $(0.027)$ & $(0.02)$ & $(0.031)$ & $(0.021)$ & $(0.027)$ & $(0.019)$ & $(0.029)$ & $(0.028)$ \\
\hline \multirow[b]{2}{*}{ CPI } & $0.321 * * *$ & & 0.145 & & $0.260 * *$ & & $0.161 *$ & & $0.240 * *$ & \\
\hline & $(0.13)$ & & $(0.125)$ & & $(0.139)$ & & $(0.133)$ & & $(0.132)$ & \\
\hline \multirow[b]{2}{*}{ Abs. Dif CPI } & & $-0.273 * * *$ & & 0.043 & & -0.068 & & $0.160 * *$ & & $-0.268 * *$ \\
\hline & & $(0.165)$ & & $(0.138)$ & & $(0.195)$ & & $(0.146)$ & & $(0.138)$ \\
\hline Adj. $\mathbf{R}^{2}$ & 0.686 & 0.677 & 0.581 & 0.575 & 0.553 & 0.553 & 0.588 & 0.599 & 0.643 & 0.648 \\
\hline $\mathbf{N}$ & 170 & 170 & 171 & 170 & 180 & 180 & 158 & 158 & 169 & 169 \\
\hline $\mathbf{F}$ & $65.102 * * *$ & $62.543 * * *$ & $41.890 * * *$ & $40.683 * * *$ & $39.343 * * *$ & $36.427 * * *$ & $39.991 * * *$ & $41.817 * * *$ & $53.550 * * *$ & $54.774 * * *$ \\
\hline
\end{tabular}


Table 1 (continued)

\begin{tabular}{|c|c|c|c|c|c|c|c|c|c|c|}
\hline & \multicolumn{2}{|c|}{ Norway } & \multicolumn{2}{|c|}{ Portugal } & \multicolumn{2}{|c|}{ Spain } & \multicolumn{2}{|c|}{ Sweden } & \multicolumn{2}{|c|}{ Switzerland } \\
\hline & Model 1 & Model 2 & Model 1 & Model 2 & Model 1 & Model 2 & Model 1 & Model 2 & Model 1 & Model 2 \\
\hline \multirow[b]{2}{*}{ Constant } & 0.421 & $8.210 * *$ & 4.45 & 8.999 & $-22.826 * * *$ & $-21.879 * * *$ & $-6.588 *$ & -0.0811 & -5.018 & -0.588 \\
\hline & $(2.832)$ & (3.086) & (7.202) & (7.191) & $(0.997)$ & $(3.723)$ & $(3.075)$ & (3.371) & $(7.951)$ & $(8.191)$ \\
\hline \multirow[b]{2}{*}{ Econ. Ties } & $0.264 * * *$ & $0.250 * * *$ & 0.02 & -0.003 & 0.138 & $0.143 * *$ & $0.215 * * *$ & $0.212 * * *$ & -0.06 & -0.057 \\
\hline & $(0.472)$ & $(0.478)$ & $(0.868)$ & $(0.831)$ & $(0.385)$ & $(0.391)$ & $(0.529)$ & $(0.529)$ & $(0.869)$ & $(0.891)$ \\
\hline \multirow[b]{2}{*}{ LogDist } & $-0.334 * * *$ & $-0.358 * * *$ & $-0.408 * *$ & $-0.404 * *$ & $-0.229 * * *$ & $-0.227 * * *$ & $-0.275 * * *$ & $-0.279 * * *$ & $-0.260 *$ & $-0.251 *$ \\
\hline & $(0.507)$ & $(0.511)$ & $(1.304)$ & $(1.295)$ & $(0.52)$ & $(0.534)$ & $(0.468)$ & $(0.467)$ & $(0.724)$ & $(0.734)$ \\
\hline \multirow[b]{2}{*}{ LOGPOP } & $0.252 * * *$ & $0.239 * * *$ & 0.188 & 0.121 & $0.623 * * *$ & $0.592 * * *$ & $0.229 * * *$ & $0.227 * * *$ & 0.132 & 0.1 \\
\hline & $(0.255)$ & $(0.257)$ & $(0.665)$ & $(0.6)$ & $(0.322)$ & $(0.323)$ & $(0.284)$ & $(0.283)$ & $(0.74)$ & $(0.709)$ \\
\hline \multirow[b]{2}{*}{ TRADE/GDP } & $-0.086 \dagger$ & $-0.102 *$ & -0.094 & -0.133 & $0.157 * *$ & $0.139 *$ & $-0.179 * * *$ & $-0.181 * * *$ & -0.161 & $-0.185^{* *}$ \\
\hline & $(0.288)$ & $(0.291)$ & $(0.653)$ & $(0.626)$ & $(0.366)$ & $(0.371)$ & $(0.319)$ & $(0.318)$ & $(0.69)$ & $(0.671)$ \\
\hline \multirow[b]{2}{*}{ Polit. Risk } & -0.088 & -0.074 & $0.286 \dagger$ & $0.291 *$ & $0.295 * *$ & $0.423 * * *$ & $0.228 * *$ & $0.226 * *$ & $0.375^{*}$ & $0.394 * *$ \\
\hline & $(0.022)$ & $(0.023)$ & $(0.047)$ & $(0.039)$ & $(0.027)$ & $(0.02)$ & $(0.026)$ & $(0.026)$ & $(0.053)$ & $(0.055)$ \\
\hline \multirow[b]{2}{*}{ CPI } & $0.543 * * *$ & & 0.088 & & $0.232 * *$ & & $0.419 * * *$ & & 0.267 & \\
\hline & $(0.097)$ & & $(0.22)$ & & $(0.123)$ & & $(0.113)$ & & $(0.249)$ & \\
\hline \multirow[b]{2}{*}{ Abs. Dif CPI } & & $-0.519 * * *$ & & -0.118 & & $-0.110 \dagger$ & & $-0.420 * * *$ & & -0.23 \\
\hline & & $(0.106)$ & & $(0.284)$ & & $(0.178)$ & & $(0.113)$ & & $(0.293)$ \\
\hline Adj. $\mathbf{R}^{2}$ & 0.665 & 0.656 & 0.29 & 0.298 & 0.561 & 0.548 & 0.698 & 0.699 & 0.43 & 0.424 \\
\hline $\mathbf{N}$ & 147 & 174 & 75 & 76 & 166 & 165 & 156 & 156 & 70 & 70 \\
\hline $\mathbf{F}$ & $60.460 * * *$ & $58.093 * * *$ & $6.527 * * *$ & $6.800 * * *$ & $37.364 * * *$ & $35.611 * * *$ & $63.430 * * *$ & $63.670 * * *$ & $10.550 * * *$ & $10.339 * * *$ \\
\hline
\end{tabular}


Table 1 (continued)

\begin{tabular}{|c|c|c|c|c|}
\hline & \multicolumn{2}{|c|}{ UK } & \multicolumn{2}{|c|}{ US } \\
\hline & Model 1 & Model 2 & Model 1 & Model 2 \\
\hline \multirow[b]{2}{*}{ Constant } & $-33.473 * * *$ & $-24.067 * * *$ & -4.811 & -2.626 \\
\hline & $(4.027)$ & $(4.49)$ & $(3.303)$ & $(3.647)$ \\
\hline \multirow[b]{2}{*}{ Econ. Ties } & $0.237 * * *$ & $0.256 * * *$ & 0.106 & 0.094 \\
\hline & $(0.515)$ & $(0.508)$ & $(0.404)$ & $(0.411)$ \\
\hline \multirow[b]{2}{*}{ LogDist } & 0.044 & 0.057 & -0.111 & -0.121 \\
\hline & $(0.515)$ & $(0.527)$ & $(0.743)$ & $(0.753)$ \\
\hline \multirow[b]{2}{*}{ LOGPOP } & $0.573 * * *$ & $0.542 * * *$ & $0.571 * * *$ & $0.553 * * *$ \\
\hline & $(0.364)$ & $(0.358)$ & $(0.253)$ & $(0.252)$ \\
\hline \multirow[b]{2}{*}{ TRADE/GDP } & 0.041 & 0.026 & $0.206 * *$ & $0.193 * * *$ \\
\hline & $(0.429)$ & $(0.43)$ & $(0.269)$ & $(0.269)$ \\
\hline \multirow[b]{2}{*}{ Polit. Risk } & 0.112 & 0.11 & 0.112 & 0.161 \\
\hline & $(0.032)$ & $(0.033)$ & $(0.02)$ & $(0.019)$ \\
\hline \multirow[b]{2}{*}{ CPI_ } & $0.487 * * *$ & & $0.276^{*}$ & \\
\hline & $(0.16)$ & & $(0.092)$ & \\
\hline \multirow[b]{2}{*}{ Abs. Dif CPI } & & $-0.478 * * *$ & & $-0.224 *$ \\
\hline & & $(0.178)$ & & $(0.131)$ \\
\hline Adj. $\mathbf{R}^{2}$ & 0.618 & 0.615 & 0.276 & 0.27 \\
\hline $\mathbf{N}$ & 157 & 156 & 159 & 158 \\
\hline $\mathbf{F}$ & $45.008 * * *$ & $44.201 * * *$ & $11.496 * * *$ & $11.131 * * *$ \\
\hline
\end{tabular}

Standardized regression coefficients are reported; standard errors are in parenthesis $p<0.10, * p<0.05, * * p<0.01$, *** $p<0.001$ 
Notes 